\title{
22. SECONDARY MINERALS IN BASALTS FROM THE MIDDLE AMERICA TRENCH, LEG 67
}

\author{
Victor B. Kurnosov, Far East Geological Institute, Vladivostok, U.S.S.R. \\ and \\ Alla Ya. Shevchenko, Institute of Oceanology, Moscow, U.S.S.R.
}

\begin{abstract}
Secondary minerals in basalts from Holes 495 and 500 include smectite and chlorite, both of which have partially replaced the basalt groundmass. In addition to these two minerals, amphibole, laumontite, albite, and a corrensitelike mineral are present in Holes 499B and 499C. Smectite, chlorite, talc, calcite, phillipsite, mica, and mixed-layer chloritemontmorillonite also fill veins in the basalts of Hole 495. The secondary mineral assemblages from Site 499 are characteristic of the initial stage of greenschist facies metamorphism.
\end{abstract}

\section{INTRODUCTION}

Deep Sea Drilling Project Leg 67 sampled basalt at Site 495, located $22 \mathrm{~km}$ seaward of the Middle America Trench axis, and at Sites 499 and 500, both located in the Trench axis. This chapter describes the secondary minerals of these rocks.

\section{METHODS}

The secondary minerals were identified mainly by X-ray diffraction analysis, supplemented by scanning electron microscope studies and wet chemical analyses. Clay minerals from the basalt groundmass were studied in bulk and also in the $\langle 1-\mu \mathrm{m}$ and 1 to $10-\mu \mathrm{m}$ fractions.

\section{SECONDARY MINERALS FROM GROUNDMASS, SITES 495 AND 500}

In the basalts from Holes 495 and 500 , groundmass glass was replaced by smectite accompanied by minor chlorite (Table 1). Trioctahedral smectites are Fe-Mgsaponites with small $\mathrm{K}$ content (Table 2 ). The clay minerals have a spongy texture (Plate 1, Figs. 1 and 2).

\section{SITE 499}

Basalts from Site 499 are highly altered. The main secondary minerals are a corrensitelike mineral (mixedlayer chlorite-smectite) and chlorite (Table 1), and these are associated with lesser amounts of amphibole and laumontite. Furthermore, plagioclases are albitized on crystal rims and along fractures.

$\mathrm{X}$-ray reflections of the corrensitelike mineral are shown in Figure 1. Nine orders from the 001, 29.2- $\AA$ reflection were identified. After saturation of the sample with ethylene glycol, the 001 reflection is $31.0 \AA$. And after heating, it is $23.6 \AA$. The mineral is trioctahedral, based on an 060 reflection of $1.54 \AA$, indicating that the chlorite is trioctahedral with a ferruginousmagnesial composition.

The texture of the sample containing the corrensitelike mineral is shown in Plate 2. Chemical analyses

\footnotetext{
${ }^{1}$ Aubouin, J., von Huene, R., et al., Init. Repts. DSDP, 67: Washington (U.S. Govt. Printing Office).
}

of the $<1-\mu \mathrm{m}$ fraction of this mineral mixed with chlorite are presented in Table 3. The corrensitelike mineral predominated in samples from Hole 499C, whereas chlorite is most abundant in the samples from Hole 499B.

Actinolite was identified by its $8.4-\AA$ reflection and the other amphibole by its $9-\AA$ reflection. Laumontite has a strong reflection at $9.47 \AA$.

\section{VEIN MINERALS}

Smectite, chlorite, talc, mica, mixed-layer chloritemontmorillonite, calcite, and phillipsite are the common vein minerals in basalts from Hole 495 (Table 4). In Sample 495-47-1, 90-92 cm an unknown mineral with an X-ray reflection at $9.66 \AA$ is also present.

Vein minerals were found in the following associations: smectite + minor chlorite \pm mica (brown clay); smectite (black clay); calcite; and calcite + phillipsite + chlorite \pm smectite. The unknown mineral and talc also occur in this last association.

The texture of smectites and iron oxides from veins in Samples 495-48-1, 80-82 cm are shown in Plate 1 (Figs. 3 and 4). Spongy textures in smectite and a euhedral phillipsite from a vesicle in Sample 500-19-1, 5-8 cm are shown in Plate 1 (Figs. 5 and 6).

Iron oxides-hydroxides also occur in vesicles in basalts from Sites 495 and 500.

\section{DISCUSSION OF RESULTS}

Study of basalts from a transect of the Middle America Trench shows alteration of basalts from Sites 495 and 500 to be similar, whereas basalts from Site 499 have different secondary mineral assemblages.

The main secondary mineral at Sites 495 and 500 in the basalt groundmass is $\mathrm{Fe}-\mathrm{Mg}$-saponite with minor chlorite. Vein minerals are smectites, chlorite, mica, mixed-layer chlorite-montmorillonite, calcite, and phillipsite. Talc was also identified in two samples. This mineral association shows that the alteration in the basalts at Sites 495 and 500 took place under alkaline and nonoxidizing conditions at temperatures about $100^{\circ} \mathrm{C}$. The presence of talc could suggest much higher temperatures up to $500^{\circ} \mathrm{C}$ (Mottl and Holland, 1978), but in the pres- 
Table 1. Secondary minerals of basalt groundmass from the Middle America Trench.

\begin{tabular}{|c|c|c|c|c|c|c|c|c|c|c|c|c|c|c|c|c|c|c|c|}
\hline \multirow{2}{*}{$\begin{array}{c}\text { Sample } \\
\text { (core-section } \\
\text { interval in } \mathrm{cm} \text { ) }\end{array}$} & \multirow{2}{*}{$\begin{array}{l}\text { Sub-bottom } \\
\text { Depth }(m)\end{array}$} & \multicolumn{3}{|c|}{ Smectite } & \multicolumn{3}{|c|}{ Chlorite } & \multicolumn{3}{|c|}{$\begin{array}{c}\text { Corrensite- } \\
\text { like Mineral }\end{array}$} & \multicolumn{3}{|c|}{ Actinolite } & \multicolumn{3}{|c|}{$\begin{array}{c}9 \dot{\AA} \\
\text { Amphibole } \\
\end{array}$} & \multicolumn{3}{|c|}{ Laumontite } \\
\hline & & 1 & 2 & 3 & 1 & 2 & 3 & 1 & 2 & 3 & 1 & 2 & 3 & 1 & 2 & 3 & 1 & 2 & 3 \\
\hline \multicolumn{20}{|l|}{ Hole 495} \\
\hline $46-1,118-120$ & 428.71 & & & $\mathrm{x}$ & & & $\mathrm{x}$ & & & & & & & & & & & & \\
\hline $46-1,121-123$ & 428.74 & & & $\mathrm{x}$ & & & & & & & & & & & & & & & \\
\hline $47-1,35-37$ & 428.85 & $\mathrm{x}$ & $\mathrm{x}$ & $\mathrm{x}$ & & & $\mathrm{x}$ & & & & & & & & & & & & \\
\hline $47-1,44-46$ & 428.94 & & & $\mathrm{x}$ & & & & & & & & & & & & & & & \\
\hline $47-1,90-92$ & 429.40 & & & $\mathrm{x}$ & & & & & & & & & & & & & & & \\
\hline $47-1,92-94$ & 429.42 & & & $\mathrm{x}$ & & & & & & & & & & & & & & & \\
\hline $48-1,80-82$ & 437.30 & $\mathrm{x}$ & $\mathrm{x}$ & $\mathrm{x}$ & & & & & & & & & & & & & & & \\
\hline $48-1,125-127$ & 437.75 & & & $\mathrm{x}$ & & & & & & & & & & & & & & & \\
\hline $48-2,52-54$ & 438.52 & & & $\mathrm{x}$ & & & $\mathrm{x}$ & & & & & & & & & & & & \\
\hline $48-3,5-7$ & 439.55 & & & $\mathrm{x}$ & & & $\mathrm{x}$ & & & & & & & & & & & & \\
\hline $48-3,47-49$ & 439.97 & & & $\mathrm{x}$ & & & & & & & & & & & & & & & \\
\hline $48-3,50-52$ & 440.00 & & & $\mathrm{x}$ & & & $\mathrm{x}$ & & & & & & & & & & & & \\
\hline $48-4,20-22$ & 441.20 & & & $\mathrm{x}$ & & & & & & & & & & & & & & & \\
\hline $48-4,55-57$ & 441.55 & $\mathrm{x}$ & $\mathrm{x}$ & $\mathrm{x}^{\mathrm{a}}$ & & & & & & & & & & & & & & & \\
\hline $48-4,57-60$ & 441.57 & $\mathrm{x}$ & $\mathrm{x}$ & $\mathrm{x}$ & & & & & & & & & & & & & & & \\
\hline $48-4,108-110$ & 442.08 & & & $\mathrm{x}$ & & & & & & & & & & & & & & & \\
\hline \multicolumn{20}{|l|}{ Hole 499B } \\
\hline $10-1,0-2$ & 297.00 & & & & $\mathrm{x}$ & $x$ & $\mathrm{x}$ & $\mathrm{x}$ & $\mathrm{x}$ & & $x$ & $\mathrm{x}$ & $\mathrm{x}$ & $\mathrm{x}$ & $x$ & $x$ & $x$ & $\mathrm{x}$ & \\
\hline $10-1,25-27$ & 297.25 & & & $\mathrm{x}$ & & & $\mathrm{x}$ & & & & & & $\mathrm{x}$ & & & $\mathrm{x}$ & & & $\mathrm{x}$ \\
\hline \multicolumn{20}{|l|}{ Hole $499 \mathrm{C}$} \\
\hline $1-1,15-17$ & 260.15 & & & & $\mathrm{x}$ & $x$ & $\mathrm{x}$ & $\mathrm{x}$ & $\mathrm{x}$ & $\mathrm{x}$ & $x$ & $x$ & & $x$ & $\mathrm{x}$ & & $x$ & $\mathrm{x}$ & \\
\hline $1-1,30-32$ & 260.30 & & & & $\mathrm{x}$ & $\mathrm{x}$ & $x$ & $\mathrm{x}$ & $x$ & $x$ & $x$ & $x$ & $x$ & $x$ & $x$ & $\mathrm{x}$ & $x$ & $\mathrm{x}$ & $x$ \\
\hline \multicolumn{20}{|l|}{ Hole 500} \\
\hline $18-1,9-11$ & 175.14 & $\mathrm{x}$ & $\mathrm{x}$ & & & & & & & & & & & & & & & & \\
\hline $18-1,18-22$ & 175.23 & $\mathrm{x}$ & $\mathrm{x}$ & $\mathrm{x}$ & & & & & & & & & & & & & & & \\
\hline $18-1,30-32$ & 175.80 & & & $\mathrm{x}$ & & & $\mathrm{x}$ & & & & & & & & & & & & \\
\hline $19-1,5-8$ & 177.55 & & & $\mathrm{x}$ & & & $\mathrm{X}$ & & & & & & & & & & & & \\
\hline $19-1,70-72$ & 178.20 & & & $x^{b}$ & & & $\mathrm{x}$ & & & & & & & & & & & & \\
\hline
\end{tabular}

Table 2. Wet chemical analyses (wt. $\%$ ) of clay minerals from the $<1$ $\mu \mathrm{m}$ fraction of basalt from the Middle America Trench, Holes 495 and 500 .

\begin{tabular}{lcr}
\hline & $\begin{array}{c}\text { Sample } \\
495-48-4,\end{array}$ \\
Component & $\begin{array}{c}\text { Sample } \\
500-18-1, \\
9-11 \mathrm{~cm}\end{array}$ \\
\hline $\mathrm{SiO}_{2}$ & 35.73 & 46.43 \\
$\mathrm{TiO}_{2}$ & 0.66 & 2.21 \\
$\mathrm{Al}_{2} \mathrm{O}_{3}$ & 12.00 & 13.99 \\
$\mathrm{Fe}_{2} \mathrm{O}_{3}$ & 9.40 & 12.62 \\
$\mathrm{FeO}$ & 2.29 & 2.73 \\
$\mathrm{MnO}$ & 0.14 & 0.26 \\
$\mathrm{MgO}$ & 7.41 & 6.57 \\
$\mathrm{CaO}$ & 5.80 & 9.77 \\
$\mathrm{Na}{ }_{2} \mathrm{O}$ & 1.88 & 1.54 \\
$\mathrm{~K}_{2} \mathrm{O}$ & 0.28 & 0.39 \\
\hline
\end{tabular}

ent case its association with calcite and phillipsite suggests formation at significantly lower temperatures.

Oxidizing conditions are indicated by the presence of iron oxides and of mica (probably celadonite). Similar conditions prevailed during alterations of basalts recovered on DSDP Legs 34 and 37 (Bass, 1976; Robinson et al., 1977; and Seyfried et al., 1976, 1978).

Higher temperature alteration, equivalent to the initial stages of greenschist facies metamorphism, is suggested by the mineral assemblage of corrensitelike miner$\mathrm{al}+$ chlorite + amphiboles + laumontite + albite, which was identified at Site 499. Some of the altering fluids in this case may have been juvenile.

Basalt metamorphism took place before deposition of the overlying sediments, because carbonaceous sediments at Sites 499,495 , and 500 show no changes in mineral composition at the contact with basalts (Kurnosov et al., this volume).

\section{ACKNOWLEDGMENTS}

We thank Dr. P. Robinson and Dr. E. Lelilov for reviewing the manuscript, and I. Kholodkevich, T. Bortina, Z. Shcheka, N. Ryapolova, and V. Piskunova for analytical work and putting the manuscript into shape.

\section{REFERENCES}

Bass, M. N., 1976. Secondary minerals in oceanic basalt, with special reference to Leg 34, Deep Sea Drilling Project. In Yeats, R. S., Hart, S. R., et al., Init. Repts. DSDP, 34: Washington (U.S. Govt. Printing Office), 393-432.

Mottl, M. J., and Holland, H. D., 1978. Chemical exchange during hydrothermal lateration of basalt by seawater I. Experimental results for major and minor components of seawater. Geochim. Cosmochim. Acta, 42:1103-1115.

Robinson, P. T., Flower, M. F. J., Schminke, H. V., and Ohnmacht, W., 1977. Low temperature alteration of oceanic basalts, DSDP Leg 37. In Aumento, F., Melson, W. G., et al., Init. Repts. DSDP, 37: Washington (U.S. Govt. Printing Office), 775-794.

Seyfried, W. E., Shanks, W. C., and Bischoff, J. L., 1976. Alteration and vein formation in Site 321 basalts. In Yeats, R. S., Hart, S. R., et al., Init. Repts. DSDP, 34: Washington (U.S. Govt. Printing Office), 351-358.

Seyfried, W. E., Shanks, W. C., and Dibble, W. E., 1978. Clay mineral formation in DSDP Leg 34 basalt. Earth Planet. Sci. Lett., 41: 265-276. 


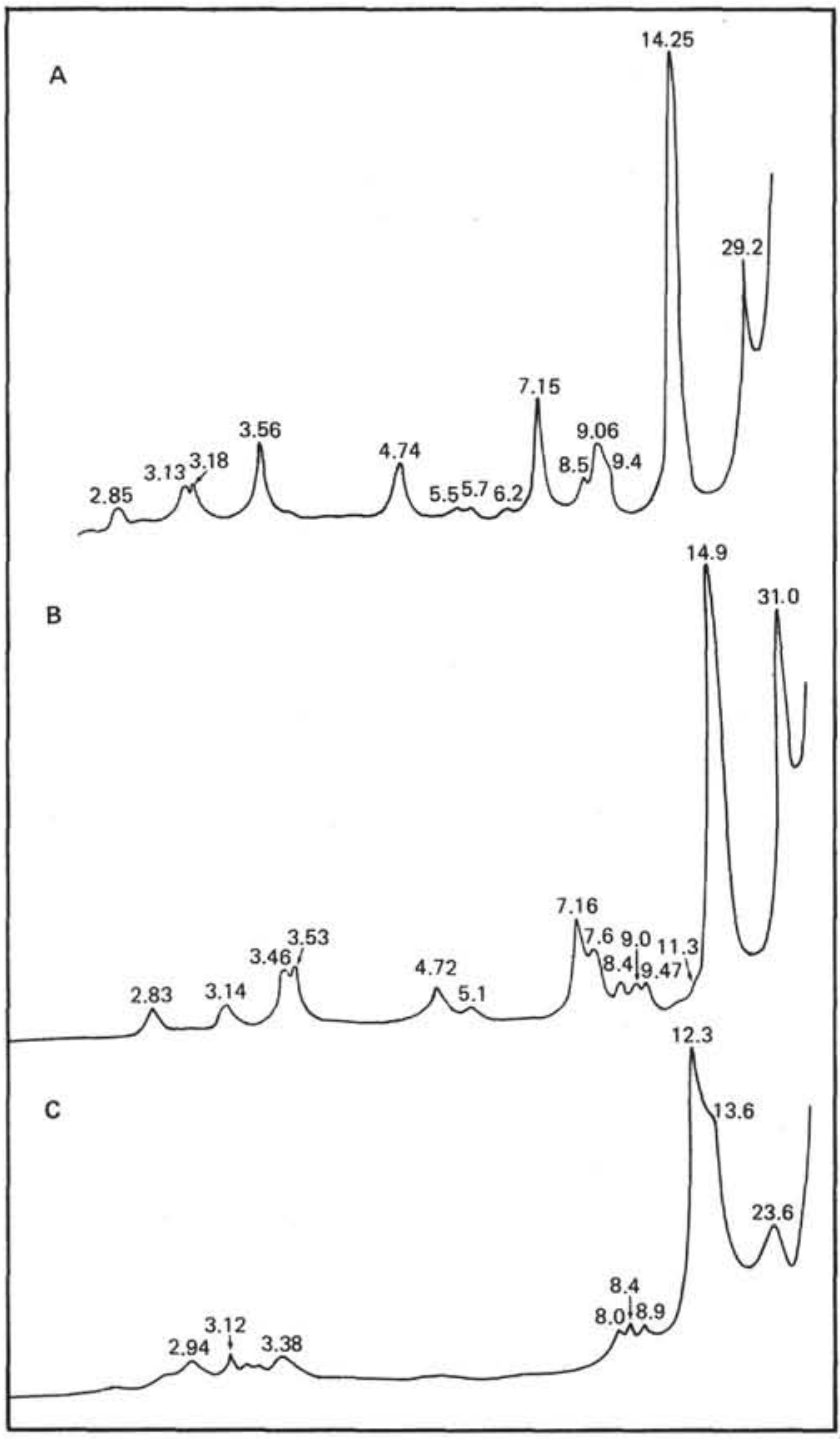

Table 3. Wet chemical analyses (wt. \%) of clay minerals from the $<1-\mu \mathrm{m}$ fraction of basalt from the Middle America Trench, Site 499.

\begin{tabular}{lrr}
\hline & $\begin{array}{c}\text { Sample } \\
499 \mathrm{C}-1-1,\end{array}$ \\
Component & $\begin{array}{c}\text { Sample } \\
499 \mathrm{~B}-10-1, \\
0-2 \mathrm{~cm}\end{array}$ \\
\hline $\mathrm{SiO}_{2}$ & 40.09 & 39.43 \\
$\mathrm{TiO}_{2}$ & 0,69 & 1.14 \\
$\mathrm{Al}_{2} \mathrm{O}_{3}$ & 14.48 & 12.85 \\
$\mathrm{Fe}_{2} \mathrm{O}_{3}$ & 9.76 & 7.28 \\
$\mathrm{FeO}$ & 3.01 & 8.55 \\
$\mathrm{MnO}$ & 0.50 & 0.11 \\
$\mathrm{MgO}$ & 15.68 & 10.70 \\
$\mathrm{CaO}$ & 3.60 & 4.63 \\
$\mathrm{Na} 2 \mathrm{O}$ & 0.81 & 1.83 \\
$\mathrm{~K}_{2} \mathrm{O}$ & 0.18 & 0.15 \\
L.o.i. & 11.54 & 12.83 \\
Total & 100.34 & 99.50 \\
\hline
\end{tabular}

Note: L.o.i. ${ }^{+}$indicates loss on ignition.

Figure 1. X-ray diffraction patterns of the $<1-\mu \mathrm{m}$ fraction with the corrensitelike mineral from Sample 499C-1-1, 15-17 cm (spacings in $\AA$ ). (A shows air-dry treatment; B, saturation with ethylene glycol; and $\mathrm{C}$, heating at $550^{\circ} \mathrm{C}$ for 1 hour.)

Table 4. Vein minerals of basalt from Hole 495 .

\begin{tabular}{|c|c|c|c|c|c|c|c|c|}
\hline $\begin{array}{c}\text { Sample } \\
\text { (core-section, } \\
\text { interval in cm) }\end{array}$ & $\begin{array}{l}\text { Sub-bottom } \\
\text { Depth (m) }\end{array}$ & Smectite & Chlorite & Talc & Mica & Calcite & Phillipsite & $\begin{array}{l}\text { Mixed-layer } \\
\text { Chlorite- } \\
\text { montmorillonite }\end{array}$ \\
\hline \multicolumn{9}{|l|}{ Hole 495} \\
\hline $47-1,35-37$ & 428.85 & $\mathrm{x}$ & $x$ & & \multirow{3}{*}{$x$} & \multirow{4}{*}{$\begin{array}{l}x \\
x \\
x\end{array}$} & & \multirow{7}{*}{$x$} \\
\hline $47-1,90-92$ & 429.40 & $\mathrm{x}$ & $x$ & & & & $\mathrm{x}$ & \\
\hline $48-1,80-82$ & 437.30 & $\mathrm{x}$ & $\mathrm{x}$ & $\mathrm{x}$ & & & $\mathrm{x}$ & \\
\hline $48-3,0-2$ & 439.50 & & & & & & & \\
\hline $48-4,20-22$ & 441.20 & $\mathrm{x}$ & $\mathrm{x}$ & & \multirow{2}{*}{$x$} & \multirow[b]{2}{*}{$x$} & \multirow[t]{2}{*}{$x$} & \\
\hline $48-4,25-27$ & 441.25 & $\mathrm{x}$ & $\mathrm{x}$ & & & & & \\
\hline $48-4,57-60$ & 441.57 & $\mathrm{x}$ & $\mathrm{x}$ & $\mathrm{x}$ & & $\mathrm{x}$ & & \\
\hline $48-4,114-116$ & 442.14 & $\hat{x}$ & & & & & & \\
\hline
\end{tabular}




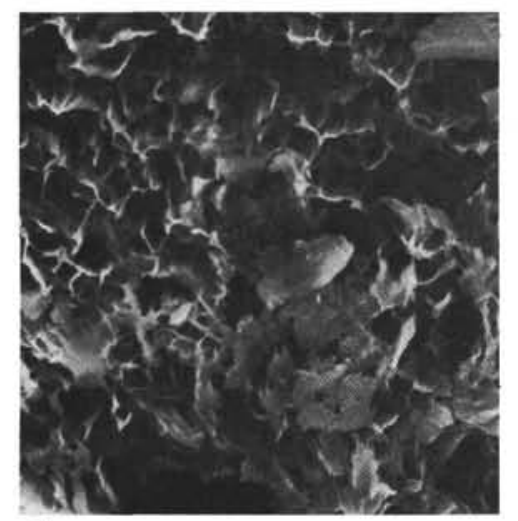

1
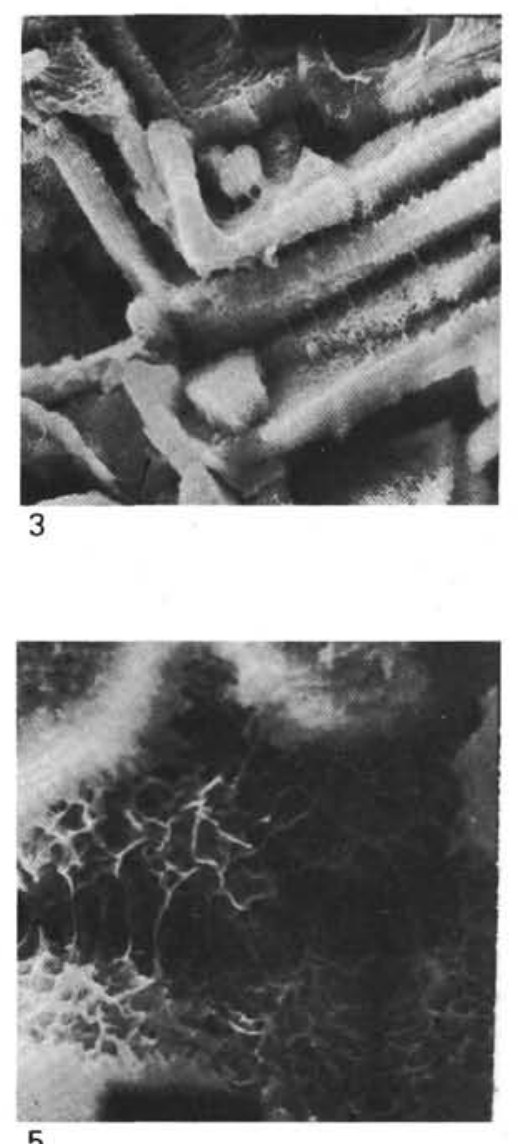

5
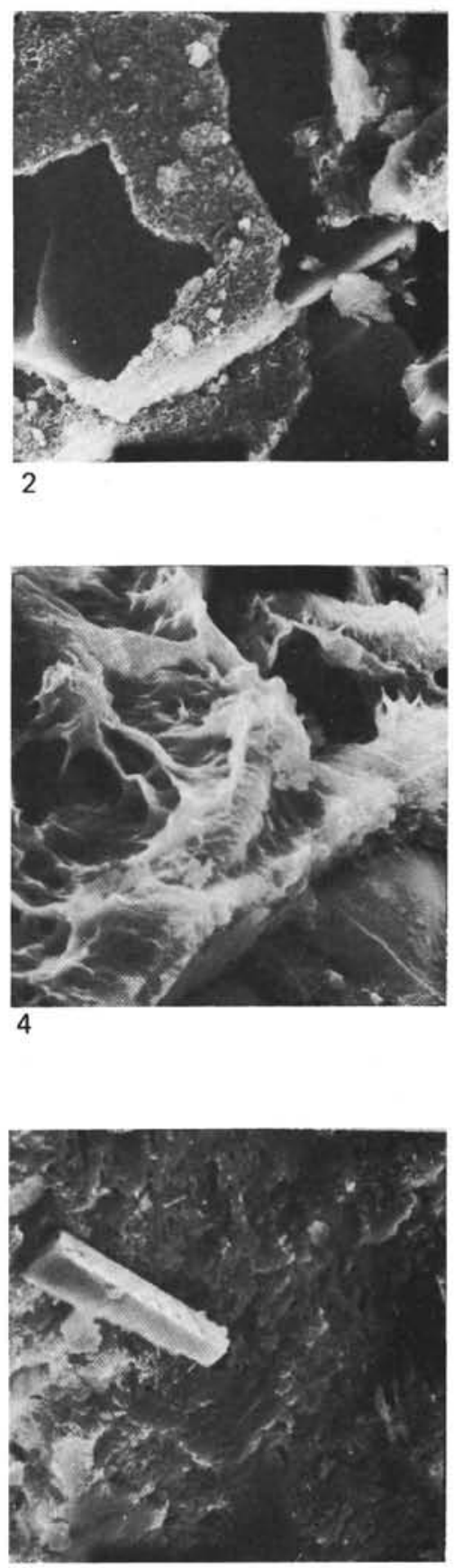

6

Plate 1. Electron photomicrographs of secondary minerals from Leg 67 basalts. 1. Smectite from basalt groundmass, $\times 750$, Sample $495-48-1$, $80-82 \mathrm{~cm}$. 2. Smectite replaced glass, $\times 500$, Sample $500-19-1,5-8 \mathrm{~cm} . \quad 3$. Smectite from vesicle, $\times 750$, Sample $495-48-1,80-82 \mathrm{~cm}$. 4. Iron hydroxide from vesicle, $\times 1000$, Sample $495-48-1,80-82 \mathrm{~cm}$. 5. Smectite from vesicle, $\times 1000$, Sample $500-19-1,5-8 \mathrm{~cm}$. 6. Phillipsite from vesicle, $\times 750$, Sample $500-19-1,5-8 \mathrm{~cm}$. 

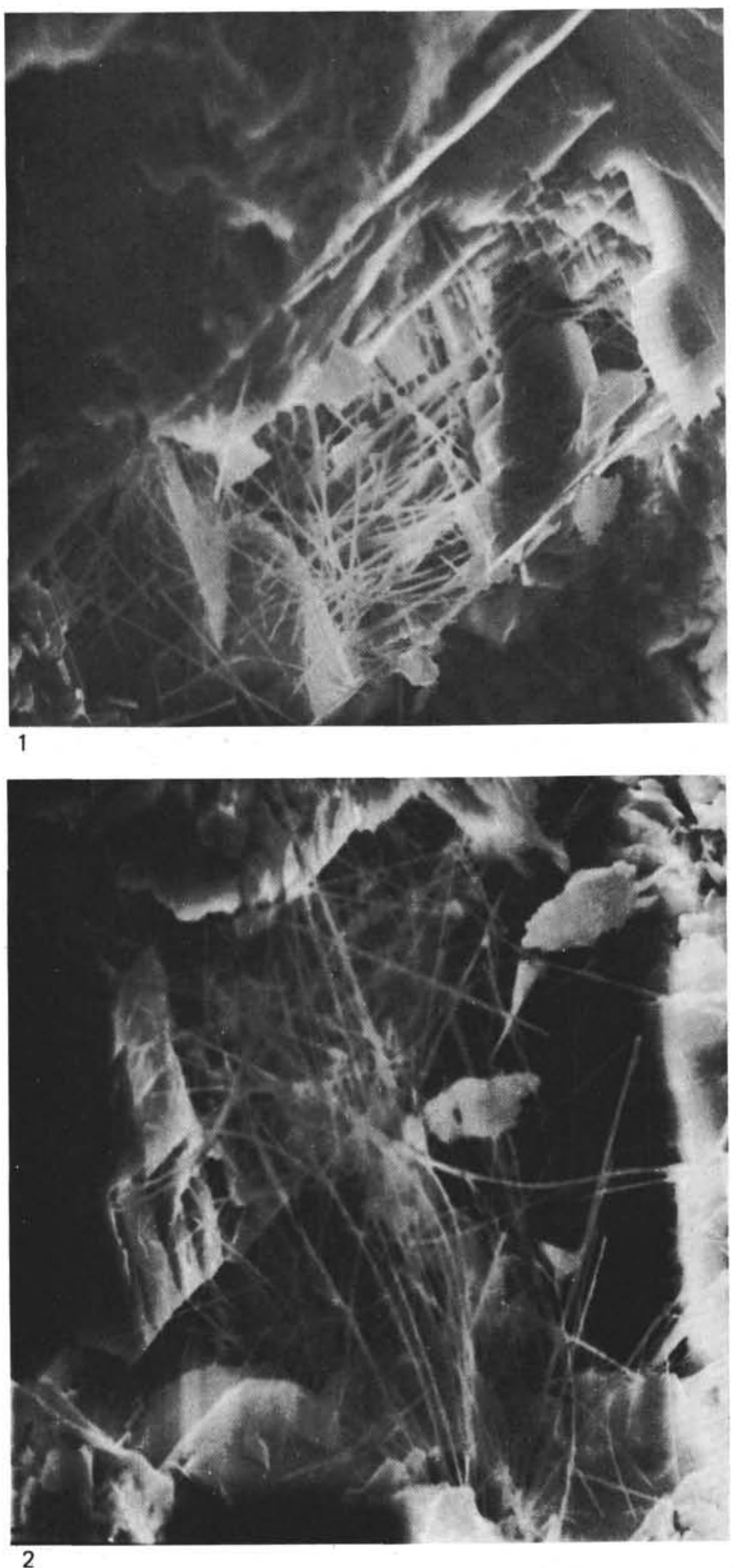

Plate 2. Electron photomicrographs of the corrensitelike mineral from Leg 67 basalt, Sample $499 \mathrm{C}-1-1,15-17 \mathrm{~cm}(1, \times 1000 ; 2, \times 3000)$. 